\title{
Blood Flow Visualization and Measurements in Microfluidic Devices Fabricated by a Micromilling Technique
}

\author{
Jaron Singhal ${ }^{1}$, Diana Pinho $2,3,{ }^{*}$, Raquel Lopes ${ }^{2}$, Patricia C. Sousa ${ }^{3}$, Valdemar Garcia $^{2}$, \\ Helmut Schütte ${ }^{1}$, Rui Lima ${ }^{2,3,4}$ and Stefan Gassmann ${ }^{1}$
}

${ }^{1}$ Jade University of Applied Science, Wilhelmshaven, Germany. ${ }^{2}$ ESTiG, Polytechnic Institute of Bragança (IPB), Portugal. ${ }^{3}$ CEFT, Faculdade de Engenharia da Universidade do Porto (FEUP), Portugal. ${ }^{4}$ Mechanical Eng. Dep., University of Minho, Campus de Azurém, 4800-058 Guimarães, Portugal

\begin{abstract}
The most common and used technique to produce microfluidic devices for biomedical applications is the soft-lithography. However, this is a high cost and time-consuming technique. Recently, manufacturers were able to produce milling tools smaller than $100 \mu \mathrm{m}$ and consequently have promoted the ability of the micromilling machines to fabricate microfluidic devices capable of performing cell separation. In this work, we show the ability of a micromilling machine to manufacture microchannels down to $30 \mu \mathrm{m}$ and also the ability of a microfluidic device to perform partial separation of red blood cells from plasma. Flow visualization and measurements were performed by using a high-speed video microscopy system. Advantages and limitations of the micromilling fabrication process are also presented.
\end{abstract}

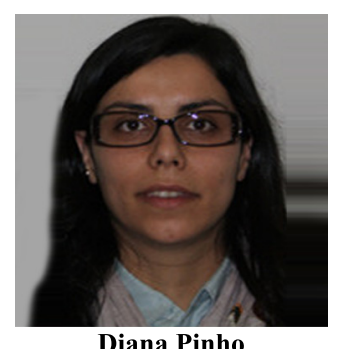

Keywords: Biomedical applications, blood cells, cell-free layer, cells separation, micromilling, microfluidic devices.

\section{INTRODUCTION}

In the field of blood analysis miniaturization, blood cells separation in microfluidic devices become an indispensable factor. In blood analysis and diagnosis, the main attention is directed towards the red blood cells (RBCs), since they exist in blood in a high concentration (typically in the range of 38$46 \%$ in women and $42-53 \%$ in men [1]) and also because they carry important information about the human's medical condition. For that reason, a lot of methods have been developed, to separate the RBCs and or white blood cells (WBCs) from the bulk blood [2-9]. To obtain a cell separation at a microfluidic level, special channel geometries have to be integrated within the microfluidic system, Fujiwara et al. [3] and Leble et al. [4] have found evidence that it is possible to create an artificial cell-free layer (CFL) in different kinds of microfluidic geometries, such as, constrictions and confluences. These in vitro studies are performed with the most popular and traditional method to fabricate microfluidic devices, the soft lithography technique, employing polydimethylsiloxane (PDMS) elastomer microchannels. By using this fabrication technique, several researchers [5-7, 9] have demonstrated that the CFL could be enhanced by using a microchannel containing a constriction followed by sudden expansion to separate plasma from the whole in vitro blood. However, the SU-8 moulds used for this method need to be fabricated by photolithography in a clean room environment with specialized equipment. For instance, a mask aligner is

*Address correspondence to this author at the Department of Mechanical Technology, ESTiG-IPB, Braganca, Portugal; Tel: +351 2733031 91; Fax: 2733130 51; E-mail: diana@ipb.pt needed for the fabrication of the masks, which are used to produce the SU-8 moulds and corresponding PDMS devices.

The acquisition of these moulds becomes very costly and time-consuming especially, for facilities without the possibility to perform photolithography. The high costs and timeconsuming involved in the traditional photolithography process are currently slowing down the interest of the industrial community to commercialize this kind of microfluidic devices for engineering and biomedical applications. Hence, it is crucial to develop simple, rapid and low-cost nonlithographic techniques to fabricate microfluidic systems.

Recently, several researchers have demonstrated that it is possible to perform cell separation by using low-cost techniques to fabricate microfluidic systems. Recently, Pinto et al. [10] have used a print-and-peel (PAP) method, known as xurography to perform blood flow studies in bifurcations and confluences. This method has shown to be an effective, novel, and rapid prototyping technique to fabricate microfluidic channels [11]. However, with this method it was not possible to fabricate microchannels less than $100 \mu \mathrm{m}$ width and as a result blood flow studies were limited to geometries bigger than $100 \mu \mathrm{m}$. Other low cost techniques such as xerographic [12], laser jet printer [13], 3D rapid prototyping technology [14] have demonstrated the ability to produce functional molds for PDMS microfluidic microdevices. Additional microfabrication methods that do not require the use of cleanrooms can be found in recent reviews performed by Rodrigues et al. [15] and Faustino et al. [16].

In this work, a nonlithographic technique is used to fabricate microchannels. A convenient option to soft-lithography is micromilling in acrylic glass (polymethyl methacrylate, 
PMMA). Recently, milling tools down to a diameter of $5 \mu \mathrm{m}$ have consequently promoted the ability of milling machines to fabricate microchannels smaller than $100 \mu \mathrm{m}$ without a clean-room facility. This technology provides a rapid production of microchannels with various geometries from the idea to the design and to the fabrication. Here, we show the ability of a micromilling machine to manufacture microchannels with geometries down to $30 \mu \mathrm{m}$. A straight contraction geometry was used in the middle of the main channel, to promote the formation of CFL adjacent to the channel walls $[3,5-7]$. Under special circumstances, like the properties of the blood sample and the dimensions of the channel geometries, the RBCs tend to migrate towards the center of the channel [17-21], surrounded by a plasma layer. The thickness of this layer depends on various factors, i.e., hematocrit (Hct) $[18,20,21]$, flow dynamics [5, 6], working fluid [17-21] and geometry of the microchannel [3-9].

The proposed geometries used in this work, have a sudden contraction to increase the CFL formation downstream and we have measured the CFL thickness at different conditions for two different geometries.

\section{MATERIALS AND METHODS}

\subsection{Fabrication of the Microfluidic Device}

The microfluidic devices tested in this study were fabricated by using a micromilling machine built for micromachining (Minitech Mini-Mill/GX). Channels were fabricated with a rectangular section inlet, $300 \mu \mathrm{m}$ in width and 40-60 $\mu \mathrm{m}$ in depth, a contraction $30 \mu \mathrm{m}$ long and two outlets. The main function of the geometries is to promote cell separation as efficient as possible using the CFL formation downstream of the contraction. Therefore, the channels are provided with narrow contractions, since previous experiments have shown that this method supports the formation of the CFL [3, 5-7]. The outlets of the channel are arranged in such way that the high concentration of RBCs should flow to Outlet 1 and the lower hematocrit should flow to Outlet 2. Fig. (1) shows an example of the fabricated channels.

Before starting the fabrication process, the geometries of the channels have to be considered, according to the metrics as well as the effect that has to be obtained and the feasibility of the fabrication has to be estimated, concerning to the limitations of the milling tools. In this work, milling tools with the diameters of $30 \mu \mathrm{m}$ and $100 \mu \mathrm{m}$ were used. In a CADSoftware geometries with defined metrics can be designed by drawing the outlines of each structure to be milled. To create the NC-code, which is readable for the milling machine, a .dxf-file containing the designed geometries is opened in VisualMill, a milling software module. By using this software several fabrication parameters, such as total cut depth, rough depth, speed and tolerance can be selected for each milling part. Note that, all parameters have to be adjusted for each milling tool used, since they have different properties, like height or fragility. The software used in this work calculates the path of the milling machine and creates a numerical code, saved in a .nc-file.

After placing the work piece on the vacuum table, the table can be adjusted by defining the reference points with the Minitech software, which provides the controlling of the machine. Special attention has to be taken for the z-axis control. The milling machine has no height control to determine the top point of the milled material. This point, which is the reference point $(z=0)$ for the milled structure has to be found manually. For this the milling tool has to be set in rotation and lowered to the material in $1 \mu \mathrm{m}$ increments. With the help of a magnifying camera, the first touch of the milling tool on the material can be observed. This process has to be repeated for each milling tool and needs to be changed manually. During the milling process, the work piece and the tool have to be cooled by a mixture of water and detergent. Otherwise, the heat resulting from the high rotation speed can damage the milling tool and the milled channels. After finishing the milling process and cleaning the device with water and detergent, the depth of the microchannels can be measured by means of a 3D optical profiler using confocal and interferometric principle (Sensofar, plu neox) [22].

The in/outlets are made of stainless steel tubes with a diameter of approximately $1 \mathrm{~mm}$ and length of $1 \mathrm{~cm}$. To obtain a better adhesion of the material, the surface around the in/outlets and the tubes can be treated by argon-plasma before adding the epoxy glue. After the curing of the glue, the device has to be cleaned in an ultrasonic bath to remove the remaining particles from the channels and also to clean the surface. Finally, the channels, Fig. (1), can be dried and sealed with an adhesive foil.

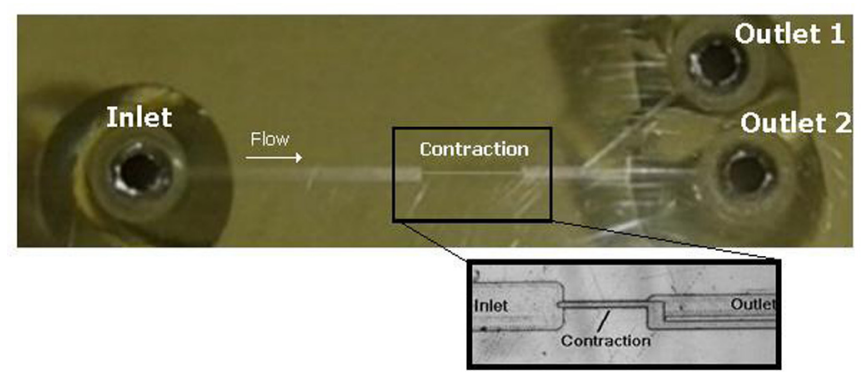

Fig. (1). Photograph of the PMMA wafer fabricated by a micromilling machine with microchannels sealed with an adhesive film and detailed optical micrograph of the contraction region.

\subsection{Working Fluid and Experimental Set-Up}

Blood was taken from a healthy sheep and EDTA had to be added to prevent coagulation of the blood cells. By centrifugation, the RBCs were extracted from the bulk blood and washed twice with saline solution. Dextran 40 (Dx40), a solution with flow properties similar to blood plasma, was used for preparing samples of 2.5 and $5(\mathrm{v} / \mathrm{v}) \%$ of Hct. All blood samples were stored hermetically at $4{ }^{\circ} \mathrm{C}$ until the experiments were performed at room temperature of approximately $20^{\circ} \mathrm{C}$.

The high-speed video microscopy system used in this study is shown in Fig. (2). This system consisted mainly of an inverted microscope (IX71, Olympus) and a high-speed camera (FASTCAM SA3, Photron). The microfluidic device was placed on the stage of the inverted microscope and a syringe pump (Harvard Apparatus PHD ULTRA) was used to produce a constant flow rate. Flow rates of 1, 5, 10, 15, 25 and $30 \mu \mathrm{L} / \mathrm{min}$ were used in the experiments. 


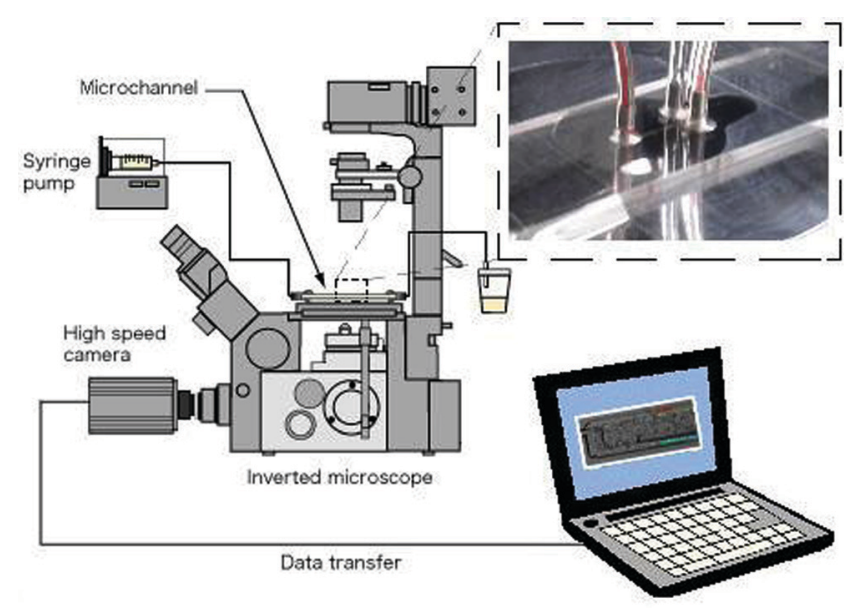

Fig. (2). High-speed video microscopy system used to perform experimental in vitro blood studies.

\subsection{Image Analysis}

In order to visualize the CFL formation in the microchannel, the images were captured around the middle of the microchannels with a resolution of $1024 \times 1024$ pixels, at a rate of 2000 frames/s. Then, all videos were evaluated using a manual image analysis by using the plugin MtrackJ [23, 24], from the software ImageJ (NIH) [25].

The CFL thickness measurements were performed by obtaining the trajectories of the RBCs flowing around the CFL boundary and subtracting them with the reference coordinates of the walls. Moreover, the coordinates resulting from these tracking's were used to determine the average thickness of the CFL (see Fig. 3). Based on the acquired data, in the form of coordinates, and according to the geometries and the flow rate, the average thickness of the CFL was evaluated.

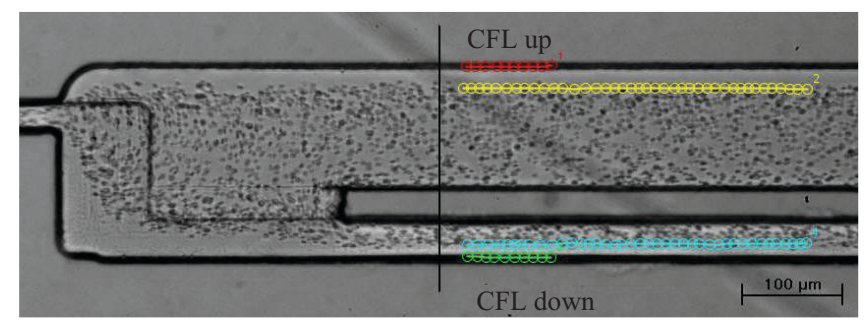

Fig. (3). Recorded experimental image showing the tracking at the channel wall and RBCs flowing around the CFL boundary. The difference between the two positions can be used to calculate the CFL thickness.

\section{RESULTS AND DISCUSSION}

\subsection{Fabrication Evaluation}

The microfluidic devices tested in this study consisted of two microchannels with a projected inlet with $300 \mu \mathrm{m}$ of width upstream the contraction. The length (L) of the contraction differs between the channels. Downstream of the sudden contraction of the microchannels has difference in the depths of the outlets. Fig. (4) shows the relevant variables of the microchannel and in Table $\mathbf{1}$ we present the corresponding dimensions.

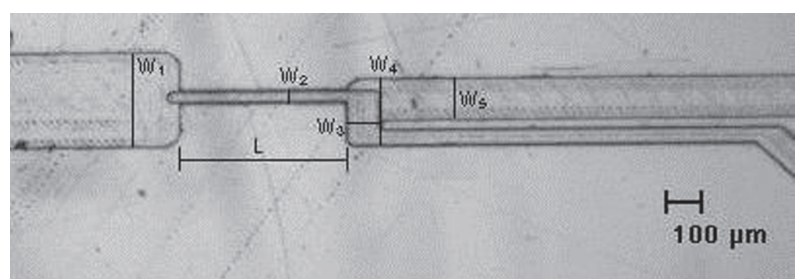

Fig. (4). A schematic illustration of the main dimensions of the tested devices.

Table 1. Main dimensions of the tested microfluidic devices.

\begin{tabular}{|c|c|c|}
\hline & Device $\mathbf{1}(\boldsymbol{\mu m})$ & Device $2(\boldsymbol{\mu m})$ \\
\hline \hline $\mathbf{W}_{\mathbf{1}}$ & 305 & 305 \\
\hline $\mathbf{L}$ & $\mathbf{1 4 5 0}$ & $\mathbf{5 1 5}$ \\
\hline $\mathbf{W}_{\mathbf{2}}$ & 30 & 30 \\
\hline $\mathbf{W}_{\mathbf{3}}$ & 300 & 300 \\
\hline $\mathbf{W}_{\mathbf{4}}$ & 205 & 205 \\
\hline $\mathbf{W}_{\mathbf{5}}$ & 130 & 130 \\
\hline
\end{tabular}

The depths of the channels were measured by using a 3D optical profiler (Sendofar, PLu neox). The measurements obtained by this profiler are presented in Fig. (5). The results have shown that the depth of Device 1, Fig. 5a), at the contraction region was $50 \mu \mathrm{m}$ and was constant for the two outlets. In contrast, the depth of Device 2 was not uniform as the depth at the contraction and outlet 2 was $40 \mu \mathrm{m}$ and at the outlet 1 the depth was $68 \mu \mathrm{m}$, Fig. 5b).

The accuracy of the fabrication, considering the original two dimensional drawing performed in the CAD Software, the real dimensions were in close agreement with the expected 2D dimensions, since the difference between both dimensions is $\pm 5 \mu \mathrm{m}$. However, it is possible to observe that the depth of the Device 2 (Fig. 5b)) is not uniform along the full length of the microchannel. Hence, the procedure to control the z-axis (height) is one of the main difficulties and challenges of this technique. More detailed information about the micromilling fabrication process can be found at Lopes et al. [26].

\subsection{Blood Flow Visualization}

Due to the high speed camera that was attached to the inverted microscope, the motion of the blood cells could be recorded with a high enough quality resolution to perform tracking measurements of individual RBCs. In this section the results of the flow visualizations are presented and discussed.

For the two tested devices in this study, the CFL thickness was measured for six different flow rates with a working fluid of $2.5 \%$ Hct. In this work, the results obtained downstream of the contraction since in the upstream part have been analyzed, only a residual formation of the CFL was observed, as shown in the Fig. (6). 
a)

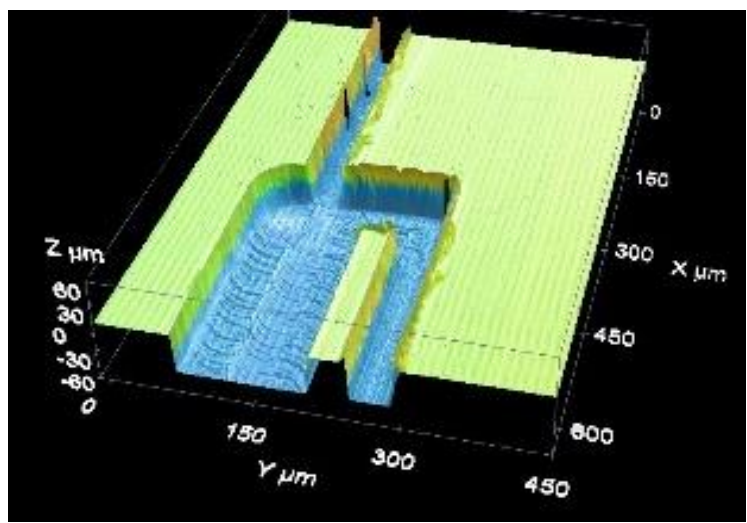

b)

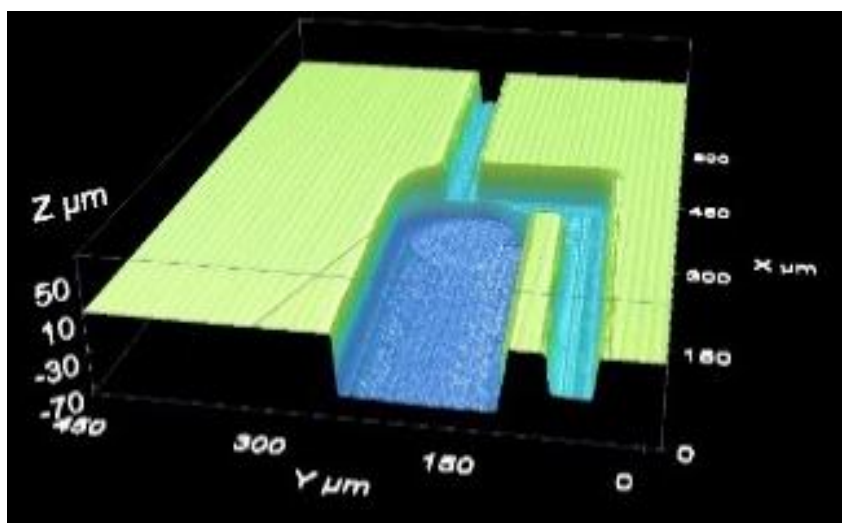

Fig. (5). Depth measurements from a 3D optical profiler: a) in Device 1 the depth is uniform and is about $50 \mu \mathrm{m}$; b) in Device 2 the depth of the contraction and outlet 2 is $40 \mu \mathrm{m}$ whereas the depth of the outlet 1 is $68 \mu \mathrm{m}$

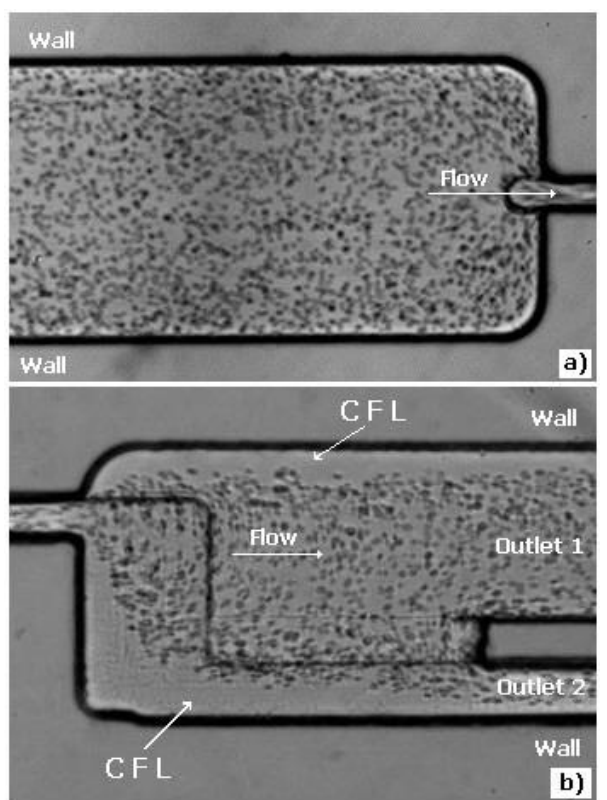

Fig. (6). In vitro blood flowing a) upstream of the contraction where there is negligible CFL; b) downstream of the contraction where there is a clear CFL.

From Fig. (7) and for the case of the Device 1, it is possible to observe that the upper CFL thickness is bigger than 20 $\mu \mathrm{m}$ and the down CFL has a thickness higher than $10 \mu \mathrm{m}$. These results show clearly that, for this particular device, the CFL thickness is asymmetric. Additionally, by increasing the flow rate, the CFL thickness slowly increases until reaching a constant value.

The ultimate purpose of this work is to obtain a full separation of RBCs from plasma. However by using the Device 1 , some amount of RBCs still flow through the outlet 2. Note that, a full separation will be obtained if all RBCs follow through the outlet 1 . In order to improve the separation efficiency a new microfluidic device (Device 2) was fabricated. In this new device, we have decided to reduce the contraction length. However, due to the difficulty of controlling the $\mathrm{z}$-axis the depth of this latter device was not uniform and different from the depth measured at the Device 2. Nevertheless, we have decided to perform in vitro blood flow measurements with the Device 2.

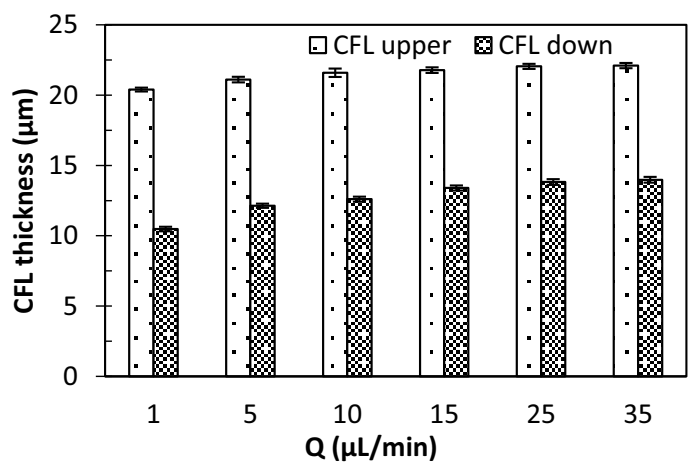

Fig. (7). CFL thickness downstream of the contraction for the Device 1 . Error bars shows a $95 \%$ of confidence interval.

The results from Fig. (8), show that by reducing the contraction length, the CFL at the upper part decreases significantly. Hence, by using this device the CFL thickness tends to have a performance more close to a symmetric behaviour. The reduction of the contraction length might be the main reason for this observed phenomenon as similar results were also observed in microfluidic devices fabricated by a softlitography technique $[5,26]$. However, in our study the increment of the depth at the outlet 1 may also have played an important role in the encountered phenomenon. Although, Device 2 may be more efficient regarding the blood separation efficiency, further research needs to be performed in the near future. Note that, by increasing the flow rate, the CFL thickness has shown a similar tendency as the one observed in the Device 1.

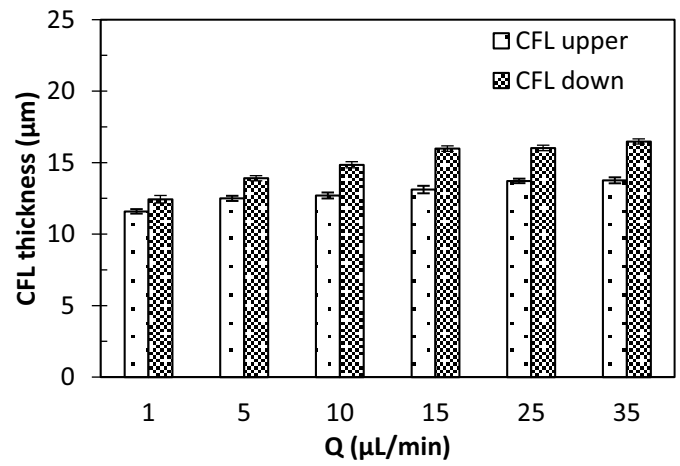

Fig. (8). CFL thickness downstream of the contraction for the Device 2 . Error bars represents a $95 \%$ confidence interval. 
For the particular case of the Device 2 in vitro blood flow measurements were also performed by using a working fluid with a Hct of $5 \%$. The results from Fig. (9) show that the CFL thickness tends to decrease by increasing the Hct. These results are in close agreement with past experimental studies [5-9]. Hence, in future work, it is crucial to take into account this last phenomenon when designing a new geometry.

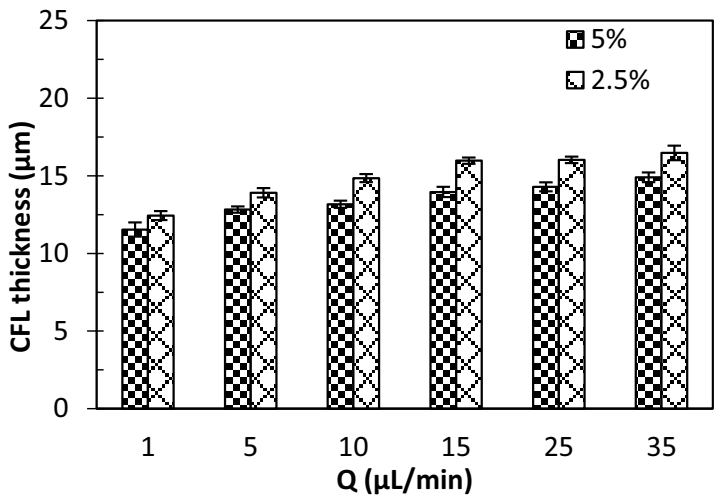

Fig. (9). CFL thickness downstream of the contraction for Device 2 at $2.5 \%$ and $5 \%$ hematocrit. Error bars represents a $95 \%$ confidence interval.

\subsection{Potential Benefits and Limitations}

Soft-lithography is the most common technique to fabricate microfluidic devices used in bioengineering and biomedical engineering. However, this technique due to the necessity of cleanrooms that increase the fabrication cost, the long time from the design to the prototype and inaccessible techniques to most biologists are slowing down the interest of the biomedical community to use this kind of devices.

In this work we have demonstrated that micromilling is a promising alternative technology to soft-lithography as lowcost microfluidic devices were developed. Overall, the results obtained from the devices produced by the micromilling technique were extremely encouraging, due to its fast fabrication time, independence from clean room facilities and ability to produce microchannels smaller than 100 $\mu \mathrm{m}$. Hence, this technique is a promising solution to manufacture affordable microfluidic devices to work in the scales required to perform blood cell analysis in continuous flow.

During our experimental activities we have observed that the produced microchannels can be used several times creating the possibility of reproducing the results. Another advantage of this technology is that the blood cells did not attached to the walls and as a result we did not observed clogging and jamming of the microchannel. However, by using PMMA we produce a microfluidic system with rigid walls, so improvements need to be done in order to have a device mimicking the in vivo environment.

Although micromilling looks promising, there are several disadvantages associated with this technique such as complex tool alignment, easily tool breakage and the roughness of the channel walls.

\section{CONCLUSIONS AND FUTURE WORK}

Two microfluidic devices were fabricated by a low cost technique known as micro-milling. Blood flow visualization and measurements of the CFL thickness were performed and parameters, such as geometry and Hct, were studied. Also, their influence in the CFL formation downstream of a contraction with a width of $30 \mu \mathrm{m}$ was analyzed. The results have demonstrated that the presence of a small contraction has promoted an improvement in the separation of the RBCs from plasma. Additionally, the results have shown that the Hct affects the separation efficiency as the CFL thickness decreases with increasing Hct.

The contraction length and the depth at the downstream part of the contraction may play an important role in the blood separation efficiency. Hence, in future work, it is important to take into account these two parameters to achieve a microfluidic device able to perform a full separation of blood cells from plasma. Overall, the results are in good agreement with studies performed in microchannels obtained by high cost fabrication techniques $[5-7,9]$. Therefore, the micromilling technique shows potential to fabricate low cost microfluidic devices able to perform blood flow studies without the use of expensive facilities such as clean-room environments

\section{CONFLICT OF INTEREST}

The authors confirm that this article content has no conflict of interest.

\section{ACKNOWLEDGEMENTS}

The authors acknowledge the financial support provided by PTDC/SAU-ENB/116929/2010 and EXPL/EMS-SIS/ 2215/2013 from FCT (Science and Technology Foundation), COMPETE, QREN and European Union (FEDER). DP acknowledge the PhD scholarship SFRH/BD/89077/2012, and P.C. Sousa acknowledges the fellowship SFRH/BPD/75258/ 2010, all attributed by FCT.

\section{REFERENCES}

[1] Thiriet, M. Biology and mechanics of blood flows. Springer, 2008, New York.

[2] Rodrigues, R.O.; Pinho, D.; Faustino, V.; Lima, R. A simple microfluidic device for the deformability assessment of blood cells in a continuous flow. Biomed. Microdevices, 2015, 17(6), 108.

[3] Fujiwara, H.; Ishikawa, T.; Lima, R. Red blood cell motions in high-hematocrit blood flowing through a stenosed microchannel. $J$. Biomech., 2009, 42, 838-843.

[4] Leble, V.; Lima, R.; Dias, R.; Fernandes, C.; Ishikawa, T.; Imai, Y.; Yamaguchi, T. Asymmetry of red blood cell motions in a microchannel with a diverging and converging bifurcation. Biomicrofluidics, 2011, 5, 044120 .

[5] Faivre, M.; Abkarian, M.; Bickraj, K. Stone HA Geometrical focusing of cells in a microfluidic device: An approach to separate blood plasma. Biorheology, 2006, 43, 147-159.

[6] Sollier, E.; Cubizolles, M.; Fouillet, Y.; Achard, J.L. Fast and continuous plasma extraction from whole human blood based on expanding cell-free layer devices. Biomed. Microdevices, 2010, 12 485-497.

[7] Pinho, D.; Yaginuma, T.; Lima, R. A microfluidic device for partial cell separation and deformability assessment. BioChip J., 2013, 7(4), 367-374.

[8] Lima, R.; Wada, S.; Tanaka, S, Takeda M, Ishikawa T, Tsubota K Imai Y, Yamaguchi $\mathrm{T}$. In vitro blood flow in a rectangular pdms microchannel: Experimental observations using a confocal micropiv system. Biomed. Microdevices, 2008, 10, 153-167.

[9] Yaginuma, T.; Oliveira, M.S.; Lima, R.; Ishikawa, T.; Yamaguchi, T. Human red blood cell behavior under homogeneous extensional 
flow in a hyperbolic-shaped microchannel. Biomicrofluidics, 2013, 7,54110 .

[10] Pinto, E.; Faustino, V.; Rodrigues, R.O.; Pinho, D.; Garcia, V.; Miranda, J.M.; Lima, R. A Rapid and Low-Cost Nonlithographic Method to Fabricate Biomedical Microdevices for Blood Flow Analysis. Micromachines, 2015, 6, 121-135.

[11] Bartholomeusz, D.A.; Boutte, R.W.; Andrade, J.D. Xurography: Rapid prototyping of microstructures using a cutting plotter. J. Microelectromech. Syst., 2005, 14, 1364-1374.

[12] Tan, A.; Rodgers, K.; Murrihy, J.; O’Mathuna, C.; Glennon, J.D. Rapid fabrication of microfluidic devices in poly(dimethylsiloxane) by photocopying. Lab Chip, 2001, 1, 7-9.

[13] Branham, M.L.; Tran-Son-Tay, R.; Schoonover, C.; Davis, P.S.; Allen, S.D.; Shyy, W. Rapid prototyping of micropatterned substrates using conventional laser printers. J. Mater. Res., 2002, 17, 1559-1562.

[14] Bonyár, A.; Sántha, H.; Ring, B.; Varga, M.; Gábor Kovács, J.; Harsányi, G. 3D rapid prototyping technology (rpt) as a powerful tool in microfluidic development. Procedia Eng. 2010, 5, 291-294.

[15] Rodrigues, R.O.; Lima, R.; Gomes, HT.; Silva, A.M.T. Polymer microfluidic devices: an overview of fabrication methods. U. Porto J. Eng., 2015, 1(1), 67-67.

[16] Faustino, V.; Catarino, S.O.; Lima, R.; Minas, G. Biomedical microfluidic devices by using low-cost fabrication techniques: a review. J. Biomech., 2016 (in press).

[17] Calejo, J.; Pinho, D.; Galindo-Rosales, F.J.; Lima, R.; CampoDeaño, L. Particulate blood analogues reproducing the erythrocytes cell-free layer in a microfluidic device containing a hyperbolic contraction. Micromachines, 2016, 7, 4.

[18] Maeda, N.; Suzuki, Y.; Tanaka, J.; Tateishi, N. Erythrocyte flow and elasticity of microvessels evaluated by marginal cell-free layer and flow resistance. Am. J. Physiol., 1996, 271, 2454-2461.
[19] Lima, R.; Ishikawa, T.; Imai, Y.; Yamaguchi, T. Blood flow behavior in microchannels: advances and future trends. In: Ricardo D.M, Rui L, Antonio A, Teresa M.M. Eds.; Single and two-phase flows on chemical and biomedical engineering. Bentham Sci., 2012, pp 513-547.

[20] Kim, S.; Ong, P.K.; Yalcin, O.; Intaglietta, M.; Johnson, P.C. The cell-free layer in microvascular blood flow. Biorheology, 2009, 46, 181-189.

[21] Garcia, V.; Dias, R.; Lima, R. In Vitro Blood Flow Behaviour in Microchannels with Simple and Complex Geometries, In: Naik, G.R. Ed.; Applied Biological Engineering - Principles and Practice. 2012, InTech, pp. 393-416.

[22] Lopes, R.; Rodrigues, R.O.; Pinho, D.; Garcia, V.; Schutte, H.; Lima, R.; Gassmann, S. Low cost microfluidic device for partial cell separation: micromilling approach. in Proceedings of 2015 IEEE International Conference on Industrial Technology, Seville, Spain.

[23] Meijering, E.; Smal, I.; Danuser, G. Tracking in molecular bioimaging. IEEE Signal Process Mag., 2006, 23, 46-53.

[24] Pinho, D.; Gayubo, F.; Pereira, A.I.; Lima, R. A comparison between a manual and automatic method to characterize red blood cell trajectories. Int. J. Num Methods Biomed. Eng., 2013, 29(9), 977-987.

[25] Abramoff, M.D.; Magalhaes, P.J.; Ram, S.J. Image Processing with Image J. Biophotonics Int., 2004, 11, 36-42.

[26] Rodrigues, R.O.; Lopes, R.; Pinho, D.; Pereira, A.I.; Garcia, V.; Gassmann, S.; Sousa, P.C.; Lima, R. In Vitro Blood Flow and CellFree Layer in Hyperbolic Microchannels: Visualizations and Measurements. BioChip J., 2016 [Epub ahead of Print]. 